\title{
Endovascular Repair of Thoracic Aortic Aneurysms
}

\author{
Laura K. Findeiss, M.D., ${ }^{1}$ and Michael E. Cody, M.D. ${ }^{1}$
}

\section{ABSTRACT}

Degenerative aneurysms of the thoracic aorta are increasing in prevalence; open repair of descending thoracic aortic aneurysms is associated with high rates of morbidity and mortality. Repair of isolated descending thoracic aortic aneurysms using stent grafts was introduced in 1995, and in an anatomically suitable subgroup of patients with thoracic aortic aneurysm, repair with endovascular stent graft provides favorable outcomes, with decreased perioperative morbidity and mortality relative to open repair. The cornerstones of successful thoracic endovascular aneurysm repair are appropriate patient selection, thorough preprocedural planning, and cautious procedural execution, the elements of which are discussed here.

KEYWORDS: Thoracic endovascular aneurysm repair (TEVAR), aneurysm, endograft, stent graft, aorta

\begin{abstract}
Objectives: Upon completion of this article, the reader should be able to state the rationale for size criteria for repair of descending thoracic aortic aneurysm, list the relative risks of perioperative morbidity and mortality with endovascular versus open repair of descending thoracic aortic aneurysm, recall elements of preprocedural planning for TEVAR, identify complications of the TEVAR procedure and understand its management strategies.

Accreditation: Tufts University School of Medicine is accredited by the Accreditation Council for Continuing Medical Education to provide continuing medical education for physicians.

Credit: Tufts University School of Medicine designates this journal-based CME activity for a maximum of 1 AMA PRA Category 1 Credit $^{T M}$. Physicians should claim only the credit commensurate with the extent of their participation in the activity.
\end{abstract}

\section{THORACIC AORTIC ANEURYSMS: EPIDEMIOLOGY AND NATURAL HISTORY}

\section{Epidemiology}

Management of thoracic aortic aneurysms is an increasingly common issue in clinical practice. An early population-based study on thoracic aortic aneurysms quoted an incidence of 5.9 per 100,000 person-years. ${ }^{1}$ More recent data on a similar population illustrates that the incidence has been rising, reaching 10.4 per 100,000 person-years. ${ }^{2}$ Although the increasing incidence of thoracic aortic aneurysms may be largely attributed to improved diagnostic capabilities given the concomitant adoption of computed tomography (CT) and transthoracic echo, the advancing age of the population at large may also contribute. In one nationwide populationbased study, the relative risk of thoracic aortic aneurysm or dissection was strongly related to age, more than doubling in each decade after age 30 until age $70 .^{3}$ The reported distribution of thoracic aortic aneurysms

\footnotetext{
${ }^{1}$ Department of Radiological Sciences, UCI Medical Center, University of California, Irvine School of Medicine, Orange, California.

Address for correspondence and reprint requests: Laura K. Findeiss, M.D., Chief of Vascular and Interventional Radiology, Department of Radiological Sciences, UCI Medical Center, University of California, Irvine School of Medicine, 101 The City Drive South, Route 140, Orange, CA 92868 (e-mail: 1findeis@uci.edu).
}

Thoracic Interventions; Guest Editor, Charles T. Burke, M.D.

Semin Intervent Radiol 2011;28:107-117. Copyright (C) 2011 by Thieme Medical Publishers, Inc., 333 Seventh Avenue, New York, NY 10001, USA. Tel: +1(212) 584-4662.

DOI: http://dx.doi.org/10.1055/s-0031-1273945.

ISSN 0739-9529. 
between males and females varies between studies, suggesting the male:female ratio may be close to $1: 1{ }^{1-4}$ An inherited pattern for thoracic aortic aneurysms has been demonstrated in $21.5 \%$ of non-Marfan syndrome patients, with an autosomal dominant pattern of inheritance with varying degree of penetrance in over threequarters of those patients. ${ }^{5}$

\section{Natural History}

The natural history of thoracic aortic aneurysm is one of sluggish growth that ultimately precipitates catastrophic complications, including dissection or rupture, often resulting in death. To offer rational treatment options for patients with thoracic aortic disease, a thorough understanding of the natural history of aneurysm growth and complications is paramount. The growth rate of thoracic aortic aneurysms varies by lesion location, with ascending aneurysms growing at a rate of $0.07 \mathrm{~cm}$ per year and descending aortic or thoracoabdominal aneurysms growing at a significantly higher rate of $0.19 \mathrm{~cm}$ per year. ${ }^{4}$ The rate of growth has also been demonstrated to increase with aneurysm size. ${ }^{6}$ The distribution of thoracic aortic aneurysms varies in different series, with aneurysms isolated to the descending aorta occurring in $22-38 \%$ of patients. ${ }^{1,2,4}$

Mortality from ruptured aneurysms approaches $100 \%$, with almost $60 \%$ of patients dying in the prehospital environment. ${ }^{7}$ Thus, rational treatment includes offering elective repair to those patients at greatest risk of rupture. It is well established that risk of aortic ruptures increases with aortic size. Aneurysms with faster growth rates are also more prone to rupture. ${ }^{8}$ Additional risk factors for rupture include aneurysms of the descending aorta and thoracoabdominal aorta, history of concomitant abdominal aortic aneurysm, history of Marfan syndrome, history of chronic obstructive lung disease, and persistent atypical pain. ${ }^{4,9}$ Interestingly, risk of rupture appears to be greater in females. ${ }^{2,4}$

\section{Surgical Treatment}

Successful resection of a fusiform aortic aneurysm with synthetic graft replacement was described as early as $1953 .{ }^{10}$ The technique has been refined over the last half century and is the gold standard in treatment of thoracic aortic aneurysms. Additional advances in graft materials, anesthesia techniques, and surgical adjuncts have undoubtedly contributed to better operative outcomes; however, mortality and morbidity nevertheless remain substantial, with the most common complications of open surgical repair being stroke, paraplegia, and renal failure. In a contemporary Medicare population study, mortality in open repair of intact descending thoracic aortic aneurysms was $12 \%$, whereas mortality involving more emergent repair of ruptured descending thoracic aorta aneurysms reached $45 \% .{ }^{11}$ A study that queried nearly 2,000 open descending thoracic aneurysm repairs over a 15-year period from the Nationwide Inpatient Sample found the mortality rate of open repair of unruptured aneurysms to be $10 \% .{ }^{12}$ Analysis of this sample revealed a complication rate of $42 \%$, including respiratory and cardiac complications, acute renal failure, and stroke and other neurologic complications. The repair of thoracic aneurysms is complex, requiring an experienced surgical and perioperative team for optimal outcomes. Mortality for repair at low volume hospitals was found to be significantly higher than at high volume hospitals $(13 \%$ and $8 \%$, respectively). It should be noted that there are high volume "centers of excellence" that are able to claim vastly improved mortality rates as low as $2.9 \%{ }^{13}$; however, such results do not appear, based on the Medicare data, to be achievable benchmarks for most institutions and do not at this time offer an accurate comparison for endovascular repair.

\section{Endovascular Repair}

Endovascular stent graft repair of descending thoracic aortic aneurysms was first described in the landmark article by Dake and colleagues ${ }^{14}$ in 1994, with ultimate U.S. Food \& Drug Administration (FDA) approval of the first commercial device for thoracic endovascular aneurysm repair (TEVAR) in 2005. Because of early recognition of markedly improved morbidity and mortality relative to open repair demonstrated in the pivotal device trial, the technology was rapidly adopted deapite the lack of data from randomized controlled trials against open repair. The lack of such trials somewhat precludes unbiased commentary relative to the two treatment options. Case control studies and comparisons to historical data have consistently indicated that outcomes following endovascular repair in the hands of experienced operators in anatomically suitable patients compare very favorably to those seen with open repair. A recent Medicare population study published this year offers insight into shifts in practice patterns and mortality in the TEVAR era. ${ }^{11}$ The authors found that 30 -day mortality for TEVAR of nonruptured aneurysms was $5.2 \%$, comparing favorably to the $12 \%$ mortality associated with open repair. Those patients undergoing TEVAR also experienced significant decrease in length of hospital and intensive care unit (ICU) stay as well as higher frequency of discharge to home. Given these benefits, it is not surprising that hospital costs associated with TEVAR of intact aneurysms were significantly decreased compared with the open cohort. In addition, the authors found that, for intact aneurysms, the frequency of TEVAR increased relative to open repair, reaching a rate of $60 \%$ of all descending thoracic aneurysm repairs by 2007 . 
In a second study evaluating real world experience strongly supportive of endovascular repair, Gopaldas et al evaluated the Nationwide Inpatient Sample data for thoracic aortic aneurysm repair, including $11,669 \mathrm{pa}^{-}$ tients during the 2 years 2006 and 2007. The group concluded that TEVAR was associated with equivalent survival, earlier discharge, more frequent discharge to home, and a $61 \%$ lower overall complication rate than open repair, but with a substantially higher cost. This finding was in a cohort of TEVAR patients that were nearly 10 years older than the open repair cohort, and who had a higher rate of comorbidities, including peripheral vascular disease, chronic kidney disease, chronic pulmonary disease, and diabetes. In-hospital mortality for both groups was low at $2.3 \% .{ }^{15}$ In a study by Greenberg et al, endovascular and surgical repair were found to have equivalent 30 -day mortality $(5.7 \%$ vs $8.3 \%$, respectively, $P=.2$ ), 12 -month mortality $(15.6 \%$ vs $15.9 \%, P=.9)$, and spinal cord ischemia (4.3\% vs $7.5 \%, P=0.08) .{ }^{16}$ In this analysis, again, it was observed that the endovascular cohort tended to be older and sicker than the open surgical cohort and it was suggested that as TEVAR becomes more commonly used in standard surgical risk patients a mortality benefit may be uncovered.

\section{Clinical Presentation}

As with abdominal aortic aneurysms, thoracic aortic aneurysms are typically asymptomatic, and may remain undetected until they dissect or rupture. Therefore, dissection and rupture remain the most common clinical presentations of TAA. A high index of suspicion is required for detection of aneurysms, but no screening protocols are recommended to date. Occasionally, penetrating atherosclerotic ulcers (PAU) or intramural hematomas, which are correlated with increased risk of dissection, may present with chest pain and stimulate an imaging workup that reveals their presence. However, in general, degenerative aneurysms are painless. Aneurysms identified in the presence of otherwise unexplained chest pain warrant immediate repair. Other symptoms may occur due to mass effect from the aneurysm, such as dysphagia or dyspnea related to compression of the trachea or bronchi or recurrent laryngeal nerve paralysis. The majority of patients present with aneurysms incidentally identified by imaging, and the decision to treat is based on aneurysm size and patient operative risk.

\section{Definitions and Indications for Treatment}

The thoracic aorta is considered aneurysmal when the diameter of the aorta is $\geq 3.5 \mathrm{~cm}$ or twice the normal diameter. Risk of rupture is proportionate to aneurysm size, ${ }^{2}$ so once an aneurysm is identified, the decision of whether to treat is based on aneurysm size and patient operative risk. Generally accepted and validated yearly risks of rupture are $0 \%, 0.3 \%, 1.7 \%$, and $3.6 \%$ for aneurysms $<4 \mathrm{~cm},>4 \mathrm{~cm},>5 \mathrm{~cm}$, and $>6 \mathrm{~cm}$, respectively. Annual risk of rupture, dissection, or death is $14.1 \%$ in patients with aneurysms larger than $6 \mathrm{~cm}$, compared with $6.5 \%$ for aneurysms between 5 and $6 \mathrm{~cm} .{ }^{17}$ Risk of rupture must be weighed against anesthesia and other perioperative risks, such that the benefit of repair outweighs the risk of the surgical procedure. As a group, the cohort of patients that present with TAA are elderly, with significant comorbidities that directly influence operative risk. Published data indicate a high incidence of hypertension (59.1\%), coronary artery disease $(27.0 \%)$, pulmonary disease $(20.9 \%)$, renal disease (13.6\%), and congestive heart failure (11.2\%) in this patient population, with a median age of 65.8 years. ${ }^{4}$ Size criteria for repair for descending aortic aneurysms are based on average surgical risk compared with annual risk of rupture, selecting the aortic size at which annual risk of rupture, dissection, or death approximates perioperative mortality. Published studies suggest a size threshold for operative repair of an isolated descending thoracic aortic aneurysm in average risk patients is in the range of $6.5 \mathrm{~cm}$, or $6.0 \mathrm{~cm}$ in patients with a family history of Marfan syndrome. ${ }^{4}$ Additionally, repair is suggested in patients with documented aneurysm growth of $>1 \mathrm{~cm}$ per year. However, specific patient characteristics must be taken into consideration prior to embarking on a plan for treatment. Some authors have advocated an approach based on "relative aortic size," whereby the risk of rupture is calculated based on risk tables that take into account the aortic size relative to the patient's body surface area. ${ }^{18}$

\section{Patient Evaluation}

The patient that presents for evaluation of thoracic aortic aneurysm may be placed into one of two general categories: 1) candidate for ongoing surveillance or 2) current potential candidate for repair.

Patients who present with aneurysms smaller than $6.0 \mathrm{~cm}$ are generally not considered to be candidates for aneurysm repair unless they have symptoms attributable to the aneurysm, or there is other evidence of impending rupture. Patients in this category should have a plan for surveillance established. The annual growth rate of descending thoracic aortic aneurysms appears to increase slightly with aneurysm size, but is on average $1.9 \mathrm{~mm}$ annually based on the largest published cohort. ${ }^{4}$ Algorithms for surveillance are dependent on an increasing growth rate with increasing size, and are somewhat dependent on individual patient characteristics. In general, noncontrast computed tomography $(\mathrm{CT})$ of the chest should be performed every 12 months until the aneurysm reaches $5.5 \mathrm{~cm}$, then every 6 months thereafter until it reaches a size appropriate for treatment. 
Patients without a family history of Marfan syndrome with aneurysms $6.5 \mathrm{~cm}$ or greater, or $6.0 \mathrm{~cm}$ or greater with a family history of Marfan syndrome, are considered potential candidates for repair and require a thorough preoperative evaluation. Critical elements of the history and physical exam include cardiac and pulmonary systems, identifying any history of coronary artery disease or chest pain, arrhythmia, congestive heart failure, chronic obstructive pulmonary disease, diabetes, or chronic kidney disease. All patients in whom repair is considered should be evaluated for surgical clearance as per hospital protocol through Cardiology, Internal Medicine, or Anesthesiology. It is important to emphasize that operative risk has not been shown to be dependent on type of anesthesia provided. There are substantial benefits to general anesthesia for this procedure and despite the temptation to consider regional anesthesia in this group of sick patients, spinal anesthesia is not considered to have a lower risk than general anesthesia overall.

Once the patient's anesthesia risk is established, and the decision to consider repair made, suitability for endovascular repair must be evaluated. Only at this point is it necessary to obtain anatomic information with CT angiography of the chest, abdomen, and pelvis.

\section{Anatomic and Associated Procedural Concerns}

After the decision is made that a patient is a reasonable candidate for repair, the decision for endovascular repair is made based on anatomic considerations. Excellent quality CT angiography including the great vessels through the femoral arteries is required for thorough evaluation and procedural planning. The following elements of the planning $\mathrm{CT}$ angiogram must be interrogated in evaluating the fitness of all candidates for placement of a thoracic aortic stent graft: (1) conformation of the aortic arch, (2) location of great vessel origins relative to the proximal margin of the aneurysm, (3) tortuosity of the descending aorta along its entire length, (4) location of mesenteric vessel origins relative to the distal margin of the aneurysm, (5) comparative diameters of proximal and distal landing zones, (6) tortuosity and diameter of the iliofemoral arteries, and (7) presence of abdominal aortic graft. In select patients in whom there is consideration of covering the origin of the left subclavian artery with the graft, the bilateral vertebral arteries and basilar artery must be evaluated as well.

\section{AORTIC ARCH}

The shape of the aortic arch has a significant impact on the rate of acute failures of thoracic endografts. ${ }^{19}$ The quality of the curvature, relationship of the curve to the origins of the left common carotid and left subclavian arteries, and acuity of the angulation of the distal arch/ proximal descending aorta are all factors in determining initial technical success and likelihood of migration, graft collapse, and type I endoleak. For purposes of placement of endovascular stent grafts, the thoracic aorta has been described in terms of zones, with zone 0 representing the ascending aorta, zone 4 representing the descending aorta, and zones 1, 2 and 3 representing the arch portion.

In an acutely angulated proximal descending aorta (zone 3), devices deployed across the apex of the curvature will not conform to the curvature unless the leading edge extends sufficiently proximally to allow the graft to lie flat in the arch segment (Fig. 1). Those grafts deployed with the leading edge just proximal to the curvature are most likely to fail due to insufficient wall apposition at the lesser curvature. Modes of failure in this setting include type I endoleak and even acute graft collapse due to the high pressure aortic blood flow causing a windsock phenomenon under the inferior margin of the proximal graft fabric. In these instances, every effort should be made to position the proximal margin of the graft just distal to the curve, or to extend proximal to the origin of the left subclavian artery, with revascularization if needed.

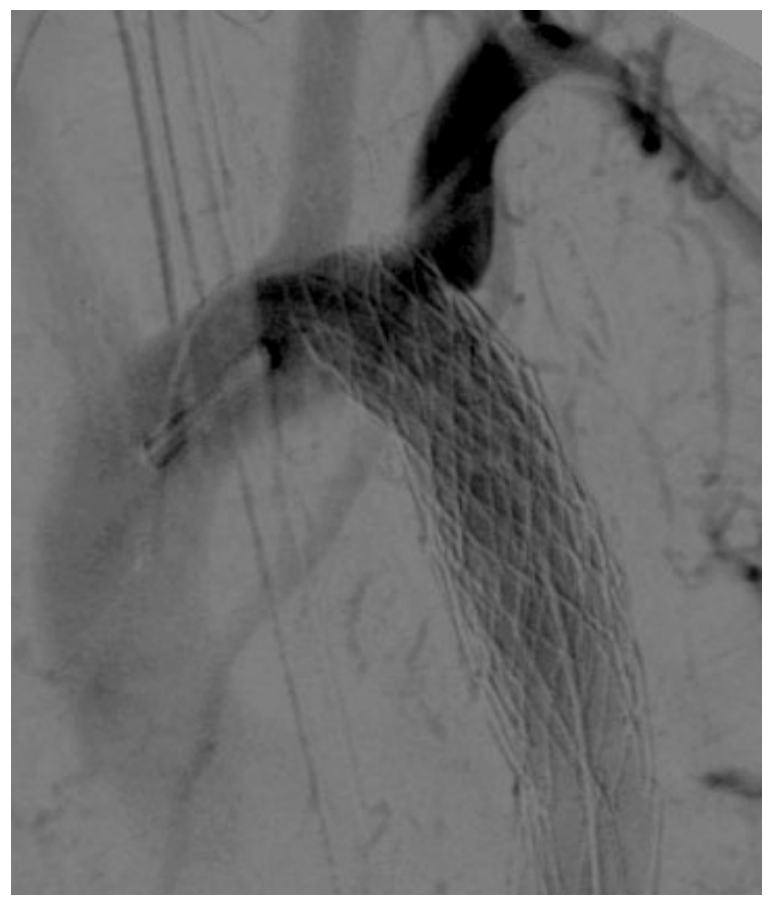

Figure 1 "Birdbeak" phenomenon. Note that the proximal graft does not conform to the lesser curvature of the aorta. This configuration may lead to collapse of the graft due to high-pressure flow dissecting under the edge of the graft material. This may be avoided by placing the proximal margin of the graft either more proximally in the horizontal portion of the arch, covering great vessel origins, or by placing distal to the arch completely in the descending aorta. 


\section{Great Vessel Origins}

The location of the origins of the great vessels must be considered relative to the proximal margin of the aneurysmal segment. If the aneurysm extends to involve the distal arch, careful attention must be paid to the lesser curvature of the aortic arch to determine the proximal extent relative to the great vessel origins. Depending on the conformation of the arch curvature, aneurysmal dilation on the lesser curve may be inadequately excluded if the graft will not conform to the curve, leading to yype I endoleak. As with unfavorable arch curvature, the graft may need to be positioned more proximally to exclude lesser curvature aneurysm, necessitating great vessel bypass or debranching (Fig. 2).

\section{Mesenteric Vessel Origins}

As with the proximal extent, the distal landing zone may be compromised based on proximity of aneurysm or tortuosity to the mesenteric arteries. The requirement for a minimum landing zone length applies to both proximal and distal extents. In patients with short distal landing zones, some operators advocate placing a catheter or wire into the celiac trunk to mark its location prior to deployment of the graft. Depending on the vascular supply to the liver, including presence of substantial gastroduodenal collateral flow or a replaced hepatic artery, the patient may tolerate coiling and overgrafting of the celiac origin. It may be important to ensure that there is patency of the inferior mesenteric artery (IMA), recognizing that the patient who is completely superior mesenteric artery (SMA) dependent is at risk of life-threatening ischemia in the event of acute compromise of the SMA. In instances requiring coverage of the celiac axis, the liver function should be monitored to evaluate for hepatic ischemia, with plans for revascularization if necessary.

\section{Comparative Diameters of Landing Zones}

Proximal and distal landing zones should not differ substantially in diameter, as it may be difficult to adequately taper stacked grafts to allow for a large differential. Each graft manufacturer has different requirements for allowable graft-to-graft size discrepancy, and there must be a prospective plan for accommodating the differential based on the individual manufacturer's instructions for use (IFU).

\section{Aortic Tortuosity}

Dilation of the aorta is accompanied by elongation, and this has a significant impact on the character of the descending aorta and aortic arch. The aorta remains relatively tethered at the diaphragmatic hiatus; therefore, elongation of the thoracic aorta leads to either bowing of the curvature of the descending aorta, or to an accordion-like compression of the elongated segment (Fig. 3). This can have significant impact on the ability to advance stiff devices from below the diaphragm into the chest, as force vectors and wire bias drive the device to push into the lateral wall of the aorta, rather than smoothly advancing to the proximal segment. Significant tortuosity should be considered a relative contraindication for all but more experienced operators.

Once the ascending aorta is accessed with a catheter, a very stiff wire advanced to the proximal arch may sufficiently straighten the tortuous segment to allow for delivery of the device. On occasion, a single stiff wire may be inadequate, and a stiff "buddy wire"
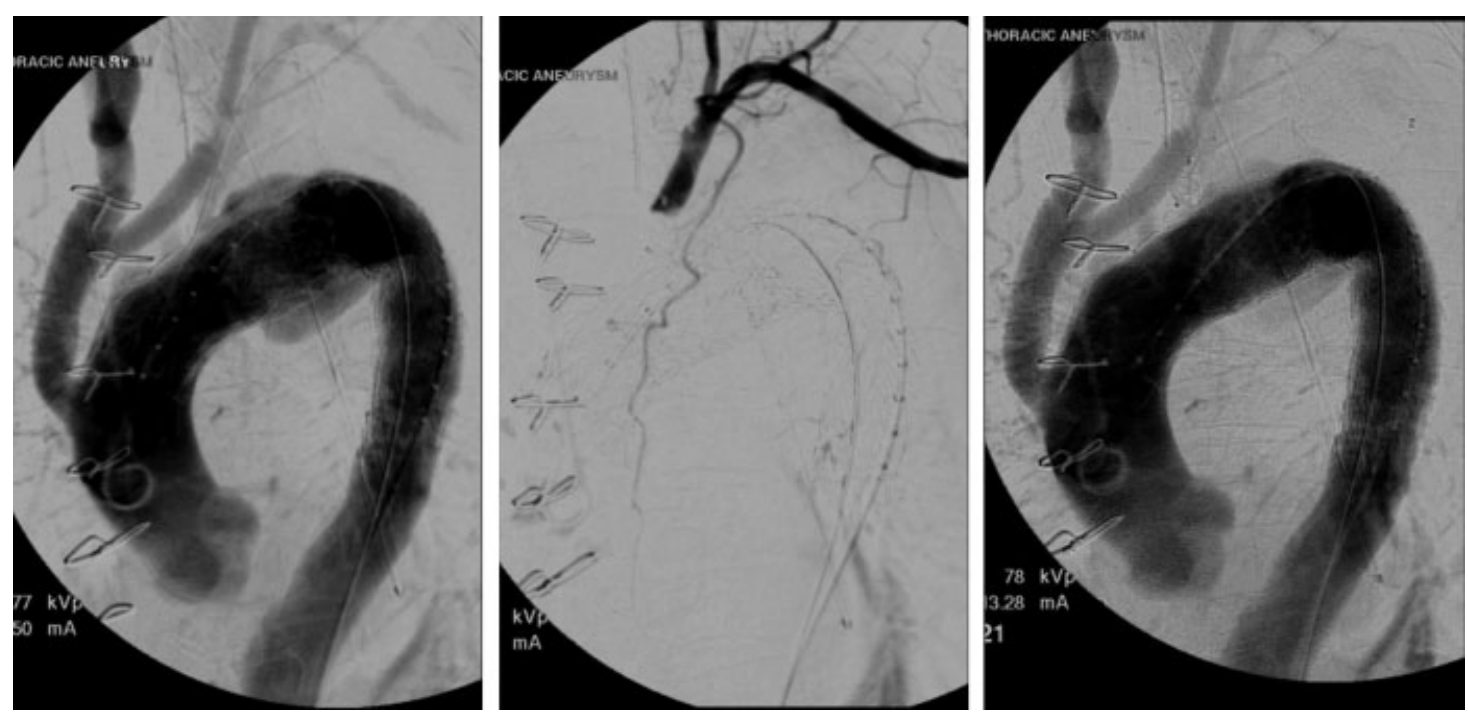

Figure 2 (A) After transposition of the left common carotid artery, the endograft was deployed just distal to the innominate origin. Note persistent endoleak. (B) After embolization of the origin of the left subclavian artery, no retrograde flow is seen from the subclavian into the aneurysm. (C) Aortogram shows persistent endoleak, likely type I. 

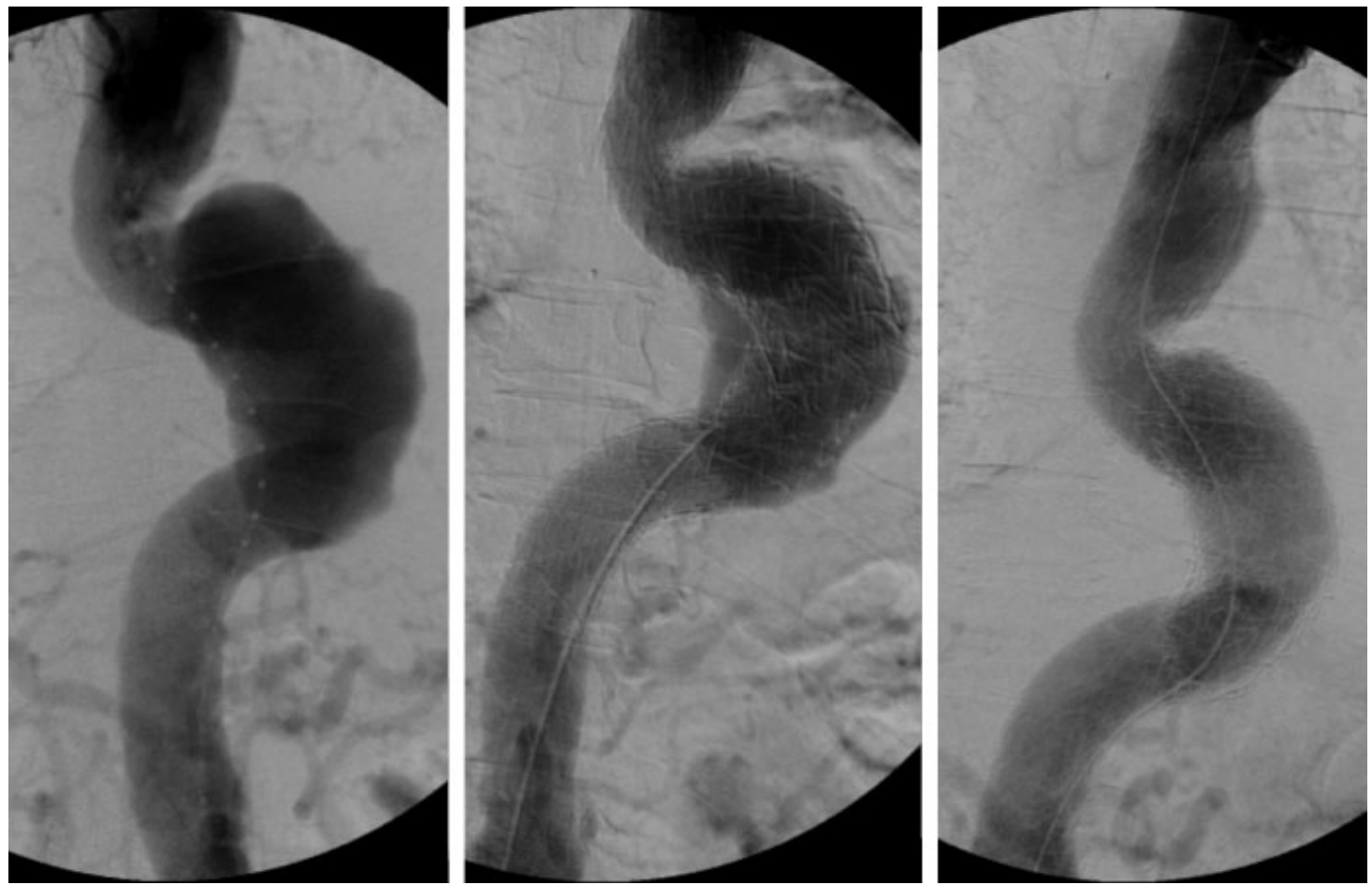

Figure 3 (A) Highly tortuous distal thoracic aorta. (B) Note lack of sufficient overlap of the proximal and distal graft components, with type III endoleak. When stacking grafts across such a segment, appropriate tube angulation is important to ensure that the graft components sufficiently overlap. (C) A bridging graft seals the endoleak.

advanced from the contralateral femoral artery may serve to further straighten the segment. On occasion, a stiff wire must be passed from the right brachial artery, through a protective catheter, into the descending aorta, and then snared from below to provide a rigid "rail" for advancement of the device.

\section{Access Vessels}

As for all catheter-mediated, image-guided procedures, obtaining safe and secure access for TEVAR is paramount, and a primary component of procedural planning. Because the profile of the thoracic endografts is significantly larger than those used for abdominal aortic endografts, there are some particular considerations beyond those applied to abdominal aortic stent grafting.

The commercially available grafts can be delivered through sheaths ranging from 20 to $25 \mathrm{~F}$, depending on the diameter of the graft. Such sheaths typically have outer diameters ranging from $22 \mathrm{~F}$ to $27 \mathrm{~F}$, or $\sim 7.3$ to $9 \mathrm{~mm}$. For safe vascular access, therefore, the access arteries must be large enough to accommodate such a sheath size.

An additional consideration in advancing large sheaths from the femoral or iliac arteries is tortuosity of the vasculature. As sheath sizes increase, so does stiffness of the delivery system, and inflexibility of the delivery systems for thoracic aortic stent grafts is a significant impediment to deployment through tortuous, athero- sclerotic arteries. As part of the procedural planning process, the iliofemoral arteries must be evaluated for tortuosity and calcification, and alternative access planned if stiff tortuous arteries are identified.

\section{Presence of Previous Abdominal Aortic Repair}

Previous open or endovascular abdominal aortic grafting appears to increase the risk of paraplegia in patients undergoing thoracic aortic endografting. In the presence of abdominal aortic graft, care should be taken to graft the absolute minimum length of thoracic aorta to treat the diseased segment and exclusion of the left subclavian artery or mesenteric arteries should be avoided or revascularization of these territories undertaken prospectively. The relative increased risk of paraplegia should be discussed with the patient, and protective measures, such as lumbar drain placement, considered.

\section{Angiographic Evaluation and Fluoroscopic Guidance}

Angiography and fluoroscopy of the aortic arch and descending thoracic aorta are the primary procedural guidance tools in placing the thoracic aortic stent graft. Therefore, the proper performance of angiography is critical. Key components of thoracic aortography include (1) high-quality imaging equipment, (2) tube angulation, 
(3) adequate contrast rate and volume, and (4) minimization of motion.

Given that endograft placement is fundamentally an image-guided procedure, it is critical that the intraprocedural imaging be of the highest possible quality. The imaging equipment used must be able to perform multiple digitally subtracted angiographic exams with steep tube angulation without tube cooling requirements. High-quality live fluoroscopy and roadmapping capabilities are critical to accurate and safe deployment of devices.

Appropriate tube angulation is required to accurately profile the origins of the great vessels. In patients requiring this procedure, the aortic arch is expected to be elongated and the descending aorta is often tortuous in addition to being aneurysmal. Choice of tube angles should be made prior to the start of the procedure based on high-quality cross-sectional imaging evaluation. Most commonly, graft deployment will be performed with the tube angulated in a very steep left anterior oblique/right posterior oblique projection, with some degree of craniocaudal tube angulation as well. The best orientation for the left subclavian origin may not be the same as for the left common carotid origin and this may require changing tube angulation several times during the procedure depending on the planned landing zone. Therefore, the table and imaging equipment must be capable of accommodating the steep angles required without compromise of the imaging, and capable of easy reorientation of the tube.

Adequately diagnostic angiography of the aortic arch relies on high contrast rate and volume, due to the large size of and high flow in the arch. Inadequate contrast bolus for angiography will cause misleading imaging with inadequate demonstration of branch vessel origins. Arch angiography should, therefore, be performed with a flush catheter at least $6 \mathrm{~F}$ in size, with contrast flow rates compatible with the patient's arch aortic diameter. Because of the high contrast load required for each angiographic run, the number of angiograms performed should be minimized through preprocedural planning and use of advanced imaging technology, including roadmapping, fluoro-fade or image overlay, etc.

A final, critical element of quality imaging for the procedure is minimization of patient motion. This can be achieved best through the use of general endotracheal anesthesia (GETA) with use of paralytics, allowing suspension of respiration during angiography for optimization of digital subtraction imaging.

\section{DEVICES}

There are three commercially manufactured, FDA-approved devices currently available. These include the TAG (WL Gore and Associates, Flagstaff, AZ), Zenith
TX2 (Cook Medical, Bloomington, IN), and Talent Thoracic (Medtronic, Santa Rosa, CA).

The construction of the TAG device mimics in large part the design of Gore's Excluder abdominal endograft. It is fabricated with an external nitinol selfexpanding stent in a spiral conformation, with an internal expanded polytetrafluoroethylene (ePTFE) graft. The graft has a characteristic scalloped, fabric-covered, barbed stent at both ends, and an ePTFE sealing cuff applied circumferentially on its outer surface at the base of each scalloped segment. The device is supplied without a sheath, and must be deployed through a $20 \mathrm{~F}$ to $24 \mathrm{~F}$ vascular access sheath, depending on graft size. The graft is deployed in the characteristic Gore graft fashion, by pulling a thread at the operator's end of the catheter to rapidly unsheath the graft. Unlike its abdominal counterpart, the graft opens from the center outwards toward both ends, rather than from the proximal to distal end. Graft diameters range from 26 to $40 \mathrm{~mm}$.

The Zenith TX 2 device is constructed similarly to the Cook Zenith abdominal endograft. A graft made of polyethylene terephthalate (Dacron; Invista Inc., Wichita, KS) is supported externally by self-expanding stainless steel barbed $\mathrm{Z}$-stents placed along the length of the graft. The device is supplied preloaded within a delivery device to be deployed through a sheath. Device sizes range from $20 \mathrm{~F}-22 \mathrm{~F}$ diameter. In contrast to the Zenith abdominal graft, proximal component grafts are covered with fabric completely, with no exposed uncovered stent at the proximal margin. The distal components contain one uncovered $Z$-stent at the distal end. The graft is delivered in a fashion similar to that used with the abdominal Zenith, with an unsheathing technique. Graft diameters range from 28 to $42 \mathrm{~mm}$.

The Medtronic Talent Thoracic endograft consists of a framework of self-expanding nitinol stents arrayed along the length of a monofilament woven polyester graft. The metallic stents are covered internally and externally by graft material with the exception of a short uncovered circumferential nitinol spring attached to the external surface of the proximal margin of the proximal graft component. An uncovered, barb-free nitinol stent forms the proximal margin of the graft designed for proximal deployment, and is available as an option at the distal end. The device is supplied preloaded within a delivery device, ranging in size from $22 \mathrm{~F}$ to $25 \mathrm{~F}$ outer diameter, that may be deployed with or without an access sheath. The delivery of the graft involves unsheathing using controls at the handle of the delivery device. Graft diameters range from 22 to $46 \mathrm{~mm}$.

\section{ACCESS}

Most commonly, the access for graft placement will be via common femoral artery cutdown after evaluation of 
the diameters and degree of tortuosity of the iliofemoral segments. As the most common complications from thoracic aortic endograft placement are related to damage to the access vessels, the importance of a conservative approach to access cannot be overemphasized. In access arteries with focal stenosis, angioplasty can be performed after placement of a stent graft within the segment to be treated. The placement of a stent graft allows aggressive focal angioplasty without risking elevation of a dissection flap upon advancing the large caliber devices.

If the common femoral, external iliac, or common iliac arteries are diffusely stenotic or very highly calcified and tortuous, a conduit is recommended for access. A length of $10-\mathrm{mm}$ diameter synthetic graft material is sutured to the most distal arterial segment of adequate diameter to accept the graft. This is most typically the distal common iliac artery, but distal abdominal aorta or even distal thoracic aorta has been used for placement of a conduit. After deployment of the graft, the conduit may be converted to a distal bypass in the setting of severe distal stenosis, or may be simply oversewn.

\section{DEVICE DEPLOYMENT}

A very stiff delivery wire should be chosen for advancing the graft through the iliac arteries and into position, and angiography of the arch should be performed with the proximal margin of the graft near the approximate position for deployment. After performance of the definitive angiogram demonstrating the great vessel origins and proximal landing zone, the location for placement of the proximal margin of the graft should be selected. Some operators advocate placing a wire via a left arm approach through the origin of the left subclavian artery to mark the origin if the graft is to be deployed distal to the subclavian. Similarly, a wire can be placed in the left common carotid origin via a right upper extremity approach to mark this vessel's location.

Manipulation of devices in the arch represents an etiology of embolic stroke during TEVAR and, therefore, movement of the graft in the arch should be minimized. In particular, some grafts require that the proximal segment be uncovered and allowed to flare with the graft proximal to the landing zone, followed by pulling the graft distally into position. When using these types of devices, the distance that the graft is repositioned after opening the proximal segment should be minimized to reduce the risk of atheroembolism.

During deployment, one hazard is migration of the graft away from the proposed landing zone. This may occur while the graft is released or uncovered, during ballooning of the graft, during removal of the delivery catheter, or with advancement of additional devices into the aorta. During deployment, mean arterial pressure (MAP) should be kept low, and many operators aim for a MAP in the range of $60-70 \mathrm{~mm} \mathrm{Hg}$.
Alternatively, pharmacologically induced asystole with adenosine has been advocated for use during initial graft deployment. The tri-lobe balloon is used commonly to allow for continued antegrade flow during ballooning. Care must be taken with removal of the delivery catheter and any other manipulation within the graft, with close fluoroscopic observation of the graft during any wire or catheter manipulation to observe for movement.

\section{SPECIAL CONSIDERATIONS}

\section{Left Subclavian Artery}

A proximal landing zone that requires coverage of the left subclavian artery (e.g., zones 0,1 , and 2) occurs in $33-41 \%$ of cases. ${ }^{20,21}$ To date, there has been much controversy regarding the need for preoperative left subclavian artery revascularization. ${ }^{22}$ Open surgical revascularization of the left subclavian artery is a relatively safe procedure, and involves a bypass originating from the left common carotid artery with ligation or embolization of the left subclavian artery origin to prevent type II endoleak. Given the lack of high-quality literature to guide management decisions in patients that will require left subclavian artery occlusion, some operators favor routine revascularization to prevent adverse outcomes that may include left arm ischemia, vertebrobasilar ischemia, spinal cord ischemia, and anterior circulation stroke. Other operators choose expectant management, with revascularization for postocclusion symptoms, or based on patient-specific anatomic considerations, such presence of left internal mammary to coronary artery bypass graft, dominant left vertebral artery, right vertebral artery occlusion, left upper extremity dialysis fistula, and in patients considered at high risk for paraplegia. An additional indication for bypass is anomalous origin of the left vertebral artery from the aorta because left arm ischemia will result from the absence of the collateral flow from the left vertebral.

An attempt to offer evidence-based guidelines for management of patients requiring left subclavian artery coverage resulted in a meta-analysis that the authors, themselves, described as being based on "very low quality evidence" consisting of observational studies. This study found that primary coverage with expectant management appeared to result in a significant increase in the risk of arm ischemia and vertebrobasilar ischemia, and a nonsignificant increase in the risk of spinal cord ischemia and anterior circulation stroke. ${ }^{23}$ Subsequent practice guidelines, issued by the Society for Vascular Surgery, ${ }^{24}$ endorse a grade 2 recommendation for routine preoperative revascularization for patients undergoing elective thoracic aortic stent graft placement with planned left subclavian artery coverage, and individualized expectant management based on anatomy, urgency, and available surgical expertise for those patients undergoing emergent 
repair. A stronger, grade 1 recommendation is made for routine revascularization in patients with anatomy that may result in ischemia to critical organs after coverage. Although these guidelines are based on all available data, it is difficult to strongly recommend routine preoperative revascularization until stronger evidence is published, and either approach in patients without anatomic or other risk factors for ischemia is supportable.

\section{Spinal Cord Ischemia}

Spinal cord ischemia (SCI) remains a dreaded complication of thoracic aortic aneurysm repair, whether operative or endovascular. Patients with neurologic deficits resulting from spinal cord ischemia experience a higher perioperative mortality rate ${ }^{25}$ than that seen in patients without evidence of SCI. Neurologic deficits secondary to thoracic aortic replacement or stent grafting tend to involve the lower trunk and lower extremities, may lateralize, and may range from minor weakness to paraplegia. In a series published in 2008 , those patients that suffered from flaccid paralysis had the most dismal outlook, with $46 \%$ perioperative mortality, $0 \%$ 5-year survival rate and limited gains in functional status, with no patients recovering the ability to walk. ${ }^{25}$ Patients that retained some degree of muscle function despite the ischemic episode were more likely to recover functional status at follow-up. ${ }^{25}$

Given the poor prospects for the cohort of patients who develop postoperative paraplegia, prospectively identifying patient-specific and procedure-specific risk factors for spinal cord ischemia remains critical. One series on open repair has found that a history of cerebrovascular events, history of renal failure, and increased age were risk factors associated with neurologic deficit (paraplegia or paraparesis). ${ }^{26} \mathrm{~A}$ system proposed by Crawford and colleagues has been traditionally used to predict complications of operative repair based on location and extent of descending thoracic aortic and thoracoabdominal aneurysms. ${ }^{27}$ Under this classification system, greater length of aorta involved confers greater risk for neurologic deficit ${ }^{28}$; however, this classification system is not as readily applicable to endovascular repair as it is to operative repair.

Because reimplantation is not an option and segmental arteries are necessarily sacrificed during endovascular repair, a new model proposed by Zoli et al shows promise in predicting risk of spinal cord ischemic complication in patients undergoing endovascular repair. ${ }^{29}$ In this model, risk of paraplegia is stratified according to the absolute number of segmental arteries sacrificed. Four subgroups were identified: fewer than 8 segmental arteries sacrificed, sacrifice of 8-12 arteries with proximal origin in the upper thorax, 8-12 segmental arteries sacrificed with proximal origin in the lower thorax, and 13 or more segmental arteries sacrificed, with paraplegia rates of $1.2 \%, 3.7 \%, 15.4 \%$, and $12.5 \%$, respectively. In addition, although data support the notion that sacrifice of segmental arteries in the T9-L2 region is correlated with spinal cord ischemia, ${ }^{30}$ the data presented by Zoli et al identifies only a limited trend toward higher spinal cord ischemia in patients with sacrifice of T11, T12, and L1. ${ }^{29}$ This article also demonstrated a relationship of neurologic deficit with sacrifice of mesenteric vessels. These findings are consistent with the notion that spinal cord perfusion is more reliant on a collateral network that includes extrasegmental arteries as opposed to a single dominant artery arising at the thoracoabdominal junction (e.g., the artery of Adamkiewicz). ${ }^{31}$

One approach with support in the literature is the preprocedural placement of an intrathecal drainage catheter via a lumbar approach to drain cerebrospinal fluid and thereby keep the intrathecal pressure low. This approach, when combined with permissive postoperative hypertension, allows for an elevated relative spinal perfusion pressure. An alternative approach is placement of a lumbar drain with the plan to lower the spinal perfusion pressure in the event that the patient develops signs of SCI or placement of a drain only in the event of symptoms. A large meta-analysis found in support of placement of lumbar drains in patients at risk for paraplegia based on aneurysm length, but advised of significant risk of subdural hematoma and meningitis. ${ }^{32}$

\section{POSTPROCEDURE MANAGEMENT}

Immediate postprocedural management considerations are related to baseline health status, vascular access, and observation for evidence of spinal cord ischemia.

Because of the overall health status and anesthesia risk in these patients, postoperative monitoring should be in an intensive care setting. Fluid balance should be carefully monitored, maintaining adequate hydration while avoiding volume overload. A Foley catheter should remain in place to allow for accurate measurement of urine output, at minimum until postoperative day 1.

In contrast to the usual approach in the patient status post open thoracic aortic repair, it is advisable to maintain MAP relatively high $(75-85 \mathrm{~mm} \mathrm{Hg})$ to improve spinal cord perfusion, as endovascular repair obviates the risk of rupture present with open repair.

Careful and frequent monitoring of lower extremity vascular and neurologic status is paramount, as early detection of vascular or neurologic complications is critical to reversing symptoms and decreasing morbidity. Should signs and symptoms of spinal cord ischemia develop, prompt evaluation by a neurologist is important, and arterial pressures should be increased to maintain a MAP in the range of $90 \mathrm{~mm} \mathrm{Hg}$ or greater. If a lumbar drain is not in place, one should be placed upon recognition of spinal cord ischemia, and the intrathecal pressure decreased to $10 \mathrm{~mm} \mathrm{Hg}$ or less. ${ }^{33}$ 
Early ambulation in this elderly and comorbid patient group is strongly recommended, as prolonged bed rest is detrimental due to rapid deconditioning in the elderly population. Many surgeons advocate ambulation within hours following open femoral artery exposure and repair. Following percutaneous repair, when feasible, we allow ambulation with assistance after 6 hours of bed rest.

\section{RISKS AND COMPLICATIONS}

The most common major adverse events in the perioperative period following thoracic aortic stent graft placement are bleeding or other vascular complication, pulmonary or cardiac complications, stroke, and paraplegia. Procedure-related mortality is substantially increased in the setting of emergency repair compared with elective repair, $5.3 \%$ versus $28 \%$, respectively, in the report of the combined EUROSTAR and UK Thoracic Endograft registries. ${ }^{34}$ The most common complications are access related, highlighting the importance of evaluating access vessels carefully. These include embolization, rupture or avulsion, or thrombosis. Rare but described late complications include retrograde type A aortic dissection, graft collapse, and aortoesophageal or aortobronchial fistula. Type A dissections appear to be more common when the presenting pathology is dissection. Endograft collapse is usually related to oversizing of the graft or placement of the proximal margin within the "danger zone" adjacent to the curvature of the arch. Registry data reveal a $4.2 \%$ rate of endoleaks present at one year in patients undergoing repair for degenerative aneurysm. ${ }^{34}$

\section{LONG-TERM SURVEILLANCE}

As with endovascular abdominal aortic aneurysm repair (EVAR), it is highly recommended that patients with thoracic aortic endografts undergo periodic imaging surveillance to evaluate for migration, endoleak, and change in size or morphology of the treated vessel. Algorithms for follow-up are not well developed; therefore, many operators follow similar schedules as those suggested for abdominal aortic endografts. CT is the preferred method of follow-up, but some authors have described the use of magnetic resonance imaging for evaluation of the thoracic aorta, as with the abdominal aorta.

When endoleaks are identified, current practice is largely guided by understanding gained from the abdominal aortic endograft literature. Type I endoleaks should be addressed when identified, with placement of proximal or distal extension cuffs as described in the EVAR population. It appears that type II endoleaks may be managed expectantly, with embolization if possible when enlargement of the aneurysm is observed. It is presumed that growing aneurysms are at risk for rupture, and if endoleak embolization is not possible, open revision is recommended.

\section{CONCLUSIONS}

Endovascular repair of descending thoracic aortic aneurysms has outcomes that are favorable in patients with suitable anatomy. It is imperative that both access and landing zone anatomy be carefully evaluated and deemed appropriate to ensure success. The most common complications of endograft placement are access related, followed by those secondary to patient comorbidities and anesthesia. Stroke and paraplegia are dreaded complications, and the rates of these complications may be diminished by careful patient selection and specific adjunctive measures. Evidence is lacking regarding the best approach to coverage of the left subclavian artery origin, but it is clear that in certain patients surgical bypass is necessary. Similarly, no evidence-based recommendations exist regarding best timetables for imaging follow-up or management of endoleaks and, currently, most operators base decisions on the EVAR data.

\section{REFERENCES}

1. Bickerstaff LK, Pairolero PC, Hollier LH, et al. Thoracic aortic aneurysms: a population-based study. Surgery 1982; 92(6):1103-1108

2. Clouse WD, Hallett JW Jr, Schaff HV, Gayari MM, Ilstrup DM, Melton LJ III. Improved prognosis of thoracic aortic aneurysms: a population-based study. JAMA 1998;280(22): 1926-1929

3. Olsson C, Thelin S, Ståhle E, Ekbom A, Granath F. Thoracic aortic aneurysm and dissection: increasing prevalence and improved outcomes reported in a nationwide population-based study of more than 14,000 cases from 1987 to 2002. Circulation 2006;114(24):26112618

4. Davies RR, Goldstein LJ, Coady MA, et al. Yearly rupture or dissection rates for thoracic aortic aneurysms: simple prediction based on size. Ann Thorac Surg 2002;73(1):17-27; discussion $27-28$

5. Albornoz G, Coady MA, Roberts M, et al. Familial thoracic aortic aneurysms and dissections-incidence, modes of inheritance, and phenotypic patterns. Ann Thorac Surg 2006;82(4): 1400-1405

6. Coady MA, Rizzo JA, Hammond GL, et al. Surgical intervention criteria for thoracic aortic aneurysms: a study of growth rates and complications. Ann Thorac Surg 1999; 67(6):1922-1926; discussion 1953-1958

7. Johansson G, Markström U, Swedenborg J. Ruptured thoracic aortic aneurysms: a study of incidence and mortality rates. J Vasc Surg 1995;21(6):985-988

8. Lobato AC, Puech-Leão P. Predictive factors for rupture of thoracoabdominal aortic aneurysm. J Vasc Surg 1998; 27(3): 446-453

9. Griepp RB, Ergin MA, Galla JD, et al. Natural history of descending thoracic and thoracoabdominal aneurysms. Ann Thorac Surg 1999;67(6):1927-1930; discussion 1953-1958 
10. De Bakey ME, Cooley DA. Successful resection of aneurysm of thoracic aorta and replacement by graft. J Am Med Assoc 1953;152(8):673-676

11. Conrad MF, Ergul EA, Patel VI, Paruchuri V, Kwolek CJ, Cambria RP. Management of diseases of the descending thoracic aorta in the endovascular era: a Medicare population study. Ann Surg 2010;252(4):603-610

12. Schermerhorn ML, Giles KA, Hamdan AD, Dalhberg SE, Hagberg R, Pomposelli F. Population-based outcomes of open descending thoracic aortic aneurysm repair. J Vasc Surg 2008;48(4):821-827

13. Achneck HE, Rizzo JA, Tranquilli M, Elefteriades JA. Safety of thoracic aortic surgery in the present era. Ann Thorac Surg 2007;84(4):1180-1185; discussion 1185

14. Dake MD, Miller DC, Semba CP, Mitchell RS, Walker PJ, Liddell RP. Transluminal placement of endovascular stentgrafts for the treatment of descending thoracic aortic aneurysms. N Engl J Med 1994;331(26):1729-1734

15. Gopaldas RR, Huh J, Dao TK, et al. Superior nationwide outcomes of endovascular versus open repair for isolated descending thoracic aortic aneurysm in 11,669 patients. J Thorac Cardiovasc Surg 2010;140(5):1001-1010

16. Greenberg RK, Lu Q Roselli EE, et al. Contemporary analysis of descending thoracic and thoracoabdominal aneurysm repair: a comparison of endovascular and open techniques. Circulation 2008;118(8):808-817

17. Elefteriades JA. Natural history of thoracic aortic aneurysms: indications for surgery, and surgical versus nonsurgical risks. Ann Thorac Surg 2002;74(5):S1877-1880; discussion S18921898

18. Davies RR, Gallo A, Coady MA, et al. Novel measurement of relative aortic size predicts rupture of thoracic aortic aneurysms. Ann Thorac Surg 2006;81(1):169-177

19. Lee WA. Failure modes of thoracic endografts: Prevention and management. J Vasc Surg 2009;49(3):792-799

20. Chiesa R, Melissano G, Marrocco-Trischitta MM, Civilini E, Setacci F. Spinal cord ischemia after elective stentgraft repair of the thoracic aorta. J Vasc Surg 2005;42(1): $11-17$

21. Feezor RJ, Martin TD, Hess PJ, et al. Risk factors for perioperative stroke during thoracic endovascular aortic repairs (TEVAR). J Endovasc Ther 2007;14(4):568-573

22. Appoo JJ, Moser WG, Fairman RM, et al. Thoracic aortic stent grafting: improving results with newer generation investigational devices. J Thorac Cardiovasc Surg 2006; 131(5):1087-1094

23. Rizvi AZ, Murad MH, Fairman RM, Erwin PJ, Montori VM. The effect of left subclavian artery coverage on morbidity and mortality in patients undergoing endovascular thoracic aortic interventions: a systematic review and metaanalysis. J Vasc Surg 2009;50(5):1159-1169

24. Matsumura JS, Lee WA, Mitchell RS, et al; Society for Vascular Surgery. The Society for Vascular Surgery Practice Guidelines: management of the left subclavian artery with thoracic endovascular aortic repair. J Vasc Surg 2009;50(5): 1155-1158

25. Conrad MF, Ye JY, Chung TK, Davison JK, Cambria RP. Spinal cord complications after thoracic aortic surgery: longterm survival and functional status varies with deficit severity. J Vasc Surg 2008;48(1):47-53

26. Safi HJ, Estrera AL, Miller CC, et al. Evolution of risk for neurologic deficit after descending and thoracoabdominal aortic repair. Ann Thorac Surg 2005;80(6):2173-2179; discussion 2179

27. Crawford ES, Crawford JL, Safi HJ, et al. Thoracoabdominal aortic aneurysms: preoperative and intraoperative factors determining immediate and long-term results of operations in 605 patients. J Vasc Surg 1986;3(3):389-404

28. Estrera AL, Miller CC III, Huynh TT, Porat E, Safi HJ. Neurologic outcome after thoracic and thoracoabdominal aortic aneurysm repair. Ann Thorac Surg 2001;72(4):12251230; discussion 1230-1231

29. Zoli S, Roder F, Etz CD, et al. Predicting the risk of paraplegia after thoracic and thoracoabdominal aneurysm repair. Ann Thorac Surg 2010;90(4):1237-1244; discussion 1245

30. Cambria RP, Davison JK, Carter C, et al. Epidural cooling for spinal cord protection during thoracoabdominal aneurysm repair: A five-year experience. J Vasc Surg 2000;31(6): 1093-1102

31. Griepp RB, Griepp EB. Spinal cord perfusion and protection during descending thoracic and thoracoabdominal aortic surgery: the collateral network concept. Ann Thorac Surg. 2007;83(2):S865-S869; discussion S890-S892

32. Cinà CS, Abouzahr L, Arena GO, Laganà A, Devereaux PJ, Farrokhyar F. Cerebrospinal fluid drainage to prevent paraplegia during thoracic and thoracoabdominal aortic aneurysm surgery: a systematic review and meta-analysis. J Vasc Surg 2004;40(1):36-44

33. Cheung AT, Pochettino A, McGarvey ML, et al. Strategies to manage paraplegia risk after endovascular stent repair of descending thoracic aortic aneurysms. Ann Thorac Surg 2005;80(4):1280-1288; discussion 1288-1289

34. Leurs LJ, Bell R, Degrieck Y, Thomas S, Hobo R, Lundbom J; EUROSTAR; UK Thoracic Endograft Registry collaborators. Endovascular treatment of thoracic aortic diseases: combined experience from the EUROSTAR and United Kingdom Thoracic Endograft registries. J Vasc Surg 2004;40(4):670679; discussion 679-680 